Because of an advertising production error, the 'Recruitment \& Events' pages in the July issues of Nature Neuroscience and Nature Reviews Neuroscience implied incorrectly that the Burnham Institute in La Jolla, California, and the Center for Neuroscience at University of California-Davis are recruiting new directors. In fact, neither position is open. The new Director for the Center for Neuroscience and Aging at The Burnham Institute is Stuart A. Lipton, and the Scientific Director of the Institute is John Reed. The Director of the Center for Neuroscience at UC Davis is Edward Jones. We apologize to all concerned for any confusion or embarrassment that this error may have caused.

\title{
Complementary localization and lateralization of orienting and motor attention
}

Matthew F. S. Rushworth, Amanda Ellison and Vincent Walsh

Nat. Neurosci. 4, 656-661 (2001)

The lettering in Figure 2 reproduced poorly. The correct figure appears below. We regret the error.

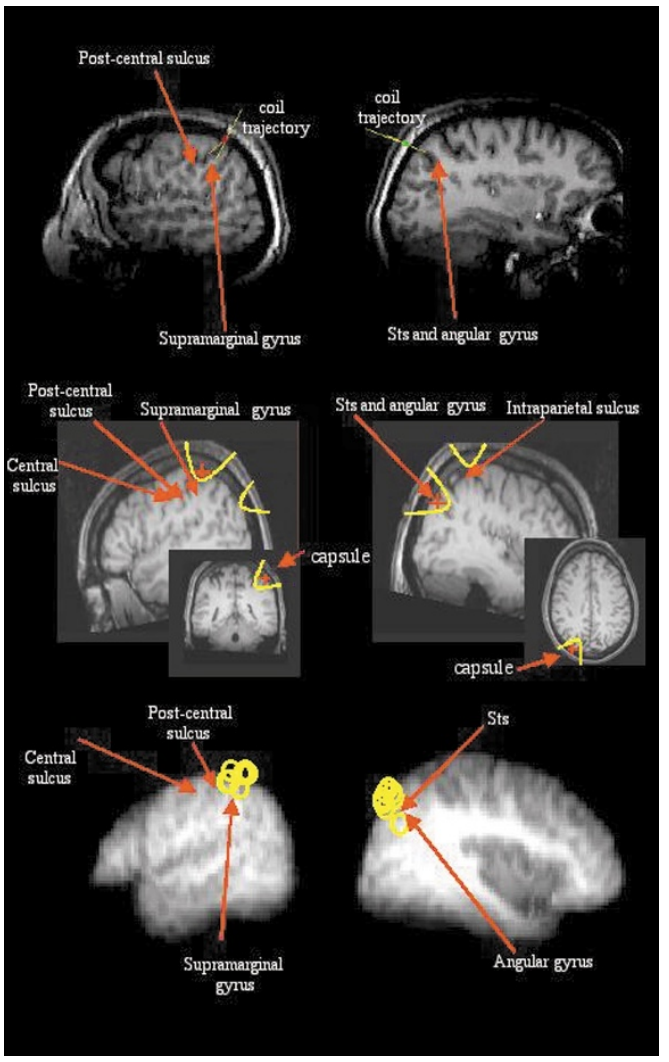

Fig. 2. TMS sites. The left SMG and right ANG sites are detailed in the left and right columns, respectively. The top row shows frameless stereotaxic recording of the TMS coil position ${ }^{14}$ during an experimental session for a single subject. The MRI brain sections are in an approximately parasagittal orientation. The red and green markers indicate the TMS coil position (outside the parasagittal section) for left SMG (left) and right ANG (right). In each case, a yellow line extends from the center of the coil, along the direction of maximum field intensity, into the underlying cortex. The middle row shows vitamin $\mathrm{E}$ capsules placed over the left SMG and right ANG sites (insets, left and right) in an MRI, taken after an experiment, and registered into standard space ${ }^{33,34}$. A red + sign is placed at the center of the underlying brain area. The main pictures show the same brain areas in sagittal sections. The main axis of the coil was in the sagittal plane at each of the positions, which were approximately $2 \mathrm{~cm}$ lateral of one another. The electrical field intensity (shown by the curved yellow lines) induced by the ANG stimulation would be reduced by a third in the sagittal plane of the SMG ${ }^{50}$. Similarly, the field induced by SMG stimulation would be reduced by a third in the sagittal plane of ANG. Bottom row, mean MRI scan of nine subjects, after registration into standard space. The sagittal sections are taken at the mean medio-lateral position of the SMG $(x=-52)$ and ANG $(x=4 I)$ sites. Yellow circles indicate the rostro-caudal and dorso-ventral positions of the centers of the stimulated brain areas in individual subjects superimposed on these sagittal planes. Although the anatomical landmarks are less clear in the averaged MRI scan, it is clear that all the SMG sites cluster between the anterior intraparietal sulcus and the post-central sulcus, and are therefore in the supramarginal gyrus. The ANG sites cluster in the angular gyrus, around the posterior, superior temporal sulcus as it runs through the angular gyrus. 